\title{
APPROXIMATE FORMULAS FOR SOME FUNCTIONS OF PRIME NUMBERS
}

\author{
Dedicated to Hans Rademacher \\ on the occasion of his seventieth birthday
}

BY

\section{J. Barkley Rosser and Lowell Schoenfeld}

\section{Acknowledgments}

The major portion of Rosser's work on this paper was done at Cornell University with support from the Air Force Office of Scientific Research. His recent work was done as an employee of the Communications Research Division (Project FOCUS) of the Institute for Defense Analyses. Schoenfeld's work was begun at the Westinghouse Research Laboratories and was completed at the Pennsylvania State University.

Three major computations were performed to obtain various of the data listed herein. The first was performed some nine years ago by workers under the direction of Mr. Marvin Howard at the Institute for Numerical Analysis, then part of the National Bureau of Standards, with support from the Office of Naval Research. The second was performed some four years ago with the cooperation of Professor R. J. Walker on the IBM 650 at the Cornell Computing Center, with support from the National Science Foundation. The third was performed recently with the cooperation of Dr. Kenneth I. Appel at the Computing Center of and with the support of Project FOCUS.

\section{Introduction}

Counting 2 as the first prime, we denote by $\pi(x), \vartheta(x)$, and $\psi(x)$, respectively, the number of primes $\leqq x$, the logarithm of the product of all primes $\leqq x$, and the logarithm of the least common multiple of all positive integers $\leqq x$; if $x<2$, we take $\pi(x)=\vartheta(x)=\psi(x)=0$. We also let $p_{n}$ denote the $n^{\text {th }}$ prime, and $\phi(n)$ denote the number of positive integers $\leqq n$ and relatively prime to $n$. Throughout, $n$ shall denote a positive integer, $p$ a prime, and $x$ a real number. We shall present approximate formulas for $\pi(x)$, $\vartheta(x), \psi(x), p_{n}, \phi(n)$, and other functions related to prime numbers.

In 1808 , on the basis of attempting to fit known values of $\pi(x)$ by an empirical formula, Legendre conjectured an approximation very similar to that given below in (2.19). In 1849, again on the basis of counts of the number of primes in various intervals, Gauss communicated to Encke a conjecture that in the neighborhood of the number $x$ the average density of the primes is $1 / \log x$. On this basis, if one should wish an estimate for the sum of $f(p)$ over all primes $p \leqq x$, the natural approximation would be

Received February 13, 1961. 


$$
\sum_{p \leqq x} f(p) \cong \int_{2}^{x} \frac{f(y) d y}{\log y} .
$$

Consequently, one would presume the following approximations:

$$
\begin{gathered}
\pi(x)=\sum_{p \leqq x} 1 \cong \int_{2}^{x} \frac{d y}{\log y}, \\
\vartheta(x)=\sum_{p \leqq x} \log p \cong \int_{2}^{x} d y \cong x, \\
\sum_{p \leqq x} \frac{1}{p} \cong \int_{2}^{x} \frac{d y}{y \log y} \cong \log \log x+B, \\
\sum_{p \leqq x} \frac{\log p}{p} \cong \int_{2}^{x} \frac{d y}{y} \cong \log x+E, \\
\prod_{\alpha<p \leqq x}\left(1-\frac{\alpha}{p}\right)=\exp \left\{\sum_{\alpha<p \leqq x} \log \left(1-\frac{\alpha}{p}\right)\right\} \\
\cong \exp \left\{c_{1}(\alpha)-\alpha \sum_{p \leqq x} \frac{1}{p}\right\} \cong \frac{c(\alpha)}{(\log x)^{\alpha}},
\end{gathered}
$$

where $\alpha$ is a real constant, usually taken to be unity.

In (2.4) we have indicated a "constant of integration," $B$, whose value is taken to be

$$
\lim _{x \rightarrow \infty}\left\{\sum_{p \leqq x} 1 / p-\log \log x\right\} .
$$

Because this limit exists, the absolute error in (2.4) tends to zero as $x$ tends to infinity. In (2.5) and (2.6), we have indicated constants $E, c_{1}(\alpha)$, and $c(\alpha)$ for analogous reasons. In (2.2) and (2.3), no constants are indicated because the limits to which they would correspond do not exist.

The validity of the approximations (2.2) through (2.6) was rigorously established just before the turn of the century by Hadamard and de la Vallee Poussin. A very excellent account of these matters is given in Ingham [6], together with extensive references to the literature.

From Ingham [6], we can get alternate expressions for $B, E$, and $c(1)$ as follows:

$$
\begin{gathered}
B=C+\sum_{p}\{\log [1-(1 / p)]+(1 / p)\} \\
E=-C-\sum_{n=2}^{\infty} \sum_{p}(\log p) / p^{n} \\
c(1)=e^{-C}
\end{gathered}
$$

where $C$ is Euler's constant. We find (2.7) and (2.9) on pp. 22-23 of Ingham [6], and can derive (2.8) from a formula near the top of p. 81. Approximate numerical values are:

$$
\begin{aligned}
& B=0.261497212847643, \\
& E=-1.332582275733221,
\end{aligned}
$$




$$
\begin{aligned}
c(1) & =0.561459483566885 \\
1 / c(1) & =1.781072417990198 \\
c(2) & =0.832429065662 .
\end{aligned}
$$

Let us define the logarithmic integral $\operatorname{li}(x)$ by

$$
\operatorname{li}(x)=\operatorname{Ei}(\log x),
$$

where $\operatorname{Ei}(y)$ is the exponential integral, defined by

$$
\operatorname{Ei}(y)=\lim _{\varepsilon \rightarrow 0+}\left\{\int_{-\infty}^{-\varepsilon} \frac{e^{t} d t}{t}+\int_{\varepsilon}^{y} \frac{e^{t} d t}{t}\right\} .
$$

Then

$$
\int_{2}^{x} \frac{d y}{\log y}=\operatorname{li}(x)-\operatorname{li}(2)
$$

Consequently, in place of (2.2), it is common to use li $(x)$ as an approximation for $\pi(x)$. This is more convenient than (2.2) because $\operatorname{Ei}(y)$ has been extensively tabulated; a convenient tabulation is given in the W.P.A. Tables [18], in which the reader should note the supplementary Table III at the end of Vol. II.

If we use two terms of the asymptotic expansion for li $(x)$, we get the following convenient approximation:

$$
\pi(x) \cong \frac{x}{\log x}\left(1+\frac{1}{\log x}\right)
$$

Using a closer approximation for $\operatorname{li}(x)$ gives the sharper result:

$$
\pi(x) \cong \frac{x}{\log x-1}
$$

From this, we see that a suitable approximation for $p_{n}$ is given by

$$
p_{n} \cong n(\log n+\log \log n-1) .
$$

Much work has been done in estimating the orders of magnitude of the errors in the various approximations listed above. A classic result appears as Theorem 23 on p. 65 of Ingham [6] in the form

$$
\pi(x)=\operatorname{li}(x)+O\left(x \exp \left\{-a(\log x)^{1 / 2}\right\}\right),
$$

where $a$ is a positive absolute constant. This means that there are positive absolute constants $a, b$, and $X$ such that for $x \geqq X$

$$
|\pi(x)-\operatorname{li}(x)|<b x \exp \left\{-a(\log x)^{1 / 2}\right\} .
$$

Improvements of this sort of result continue to appear. The sharpest known, given in Vinogradov [17], is

$$
\pi(x)=\operatorname{li}(x)+O\left(x \exp \left\{-a(\log x)^{3 / 5}\right\}\right) .
$$


Undoubtedly this is not the best possible result, but the precise behavior of $\pi(x)-\operatorname{li}(x)$ depends on the location of the zeros of the Riemann zeta function, and cannot be determined until we have more precise information about them than we have now. A discussion of this point appears in Chap. IV of Ingham [6].

Even in our present ignorance about the zeros of the zeta function, it can be shown that li $(x)$ alone cannot be a wholly satisfactory approximation to $\pi(x)$. Specifically, it has been shown that there is a sequence of values of $x$, tending to infinity, at which alternately

and

$$
\pi(x)-\operatorname{li}(x)>x^{1 / 2} / \log x
$$

$$
\pi(x)-\operatorname{li}(x)<-x^{1 / 2} / \log x .
$$

Indeed, Theorem 35 on p. 103 of Ingham [6] states a significantly stronger result. An analogous result for $\vartheta(x)-x$ follows from Theorem 34 on $p$. 100 of Ingham [6] by means of the relations

$$
\begin{gathered}
\psi(x)=\sum_{n=1}^{\infty} \vartheta\left(x^{1 / n}\right), \\
\vartheta(x)=\sum_{n=1}^{\infty} \mu(n) \psi\left(x^{1 / n}\right) .
\end{gathered}
$$

Each of these summations is in fact only finite, since the summands become zero as soon as $n>(\log x) /(\log 2)$. The first of these equations is derived on p. 12 of Ingham [6], and inversion of the first gives the second, in which $\mu$ is the Möbius function defined on p. 567 of Landau [7], vol. 2.

Once one has a good estimate for $\pi(x)$ - li $(x)$, one can get an approximation for sums of functions of primes as follows. Using the Stieltjes integral, one has

$$
\sum_{p \leqq x} f(p)=\int_{2-}^{x} f(y) d \pi(y) .
$$

Integration by parts gives

$$
\begin{aligned}
\sum_{p \leqq x} f(p) & =f(x) \pi(x)-\int_{2}^{x} f^{\prime}(y) \pi(y) d y \\
& =f(x) \pi(x)-\int_{2}^{x} f^{\prime}(y) \operatorname{li}(y) d y-\int_{2}^{x} f^{\prime}(y)\{\pi(y)-\operatorname{li}(y)\} d y .
\end{aligned}
$$

Then integration by parts gives

$$
\begin{aligned}
\sum_{p \leqq x} f(p)= & \int_{2}^{x} \frac{f(y) d y}{\log y}+f(2) \operatorname{li}(2) \\
& +f(x)\{\pi(x)-\operatorname{li}(x)\}-\int_{2}^{x} f^{\prime}(y)\{\pi(y)-\operatorname{li}(y)\} d y
\end{aligned}
$$

which is the precise version of (2.1). If the integral in (2.28) below con- 
verges, we can rewrite $(2.26)$ as

$$
\begin{aligned}
\sum_{p \leqq x} f(p)= & \int_{2}^{x} \frac{f(y) d y}{\log y}+K_{f} \\
& +f(x)\{\pi(x)-\operatorname{li}(x)\}+\int_{x}^{\infty} f^{\prime}(y)\{\pi(y)-\operatorname{li}(y)\} d y
\end{aligned}
$$

where $K_{f}$ is the constant given by

$$
K_{f}=f(2) \operatorname{li}(2)-\int_{2}^{\infty} f^{\prime}(y)\{\pi(y)-\operatorname{li}(y)\} d y .
$$

Using these, we can get sharper forms of (2.3) through (2.6). Thus, from (2.21) and (2.26), we get

$$
\vartheta(x)=x+O\left(x \exp \left\{-a(\log x)^{1 / 2}\right\}\right) .
$$

From (2.21) and (2.27), we get

$$
\begin{gathered}
\sum_{p \leqq x} 1 / p=\log \log x+B+O\left(\exp \left\{-a(\log x)^{1 / 2}\right\}\right), \\
\sum_{p \leqq x}(\log p) / p=\log x+E+O\left(\exp \left\{-a(\log x)^{1 / 2}\right\}\right) .
\end{gathered}
$$

From (2.30) we proceed as in (2.6) to get

$$
\prod_{\alpha<p \leqq x}\left(1-\frac{\alpha}{p}\right)=\frac{c(\alpha)}{(\log x)^{\alpha}}+O\left(\exp \left\{-a(\log x)^{1 / 2}\right\}\right) .
$$

From (2.29) and (2.24), one can get a formula for $\psi(x)$ analogous to (2.29). By starting from (2.23) rather than (2.21), one can get even sharper results than (2.29) through (2.32).

Though results like those above are interesting, and are difficult to prove, they are of little use for getting dependable numerical approximations unless values of $a, b$, and $X$ in (2.22) are furnished; this is seldom done. In Rosser [12], explicit bounds were presented for the errors in our approximations. More recently, much better bounds have been obtained by using modern computing machinery and taking advantage of new information about the zeros of the zeta function. These results will be stated in the early part of the present paper, with the proofs being mainly withheld until the later sections.

\section{Widely applicable approximations}

For a very sharp approximation, one must either use complicated formulas or be satisfied with validity over a limited range. In this section, we shall list approximations which combine the advantages of being reasonably simple, reasonably precise, and valid for nearly all values. Note that Theorem 1 below will replace (2.18) by closely related and specific inequalities, while Theorems 2-7 will do the same for $(2.19),(2.20),(2.3),(2.4),(2.5)$, and (2.6) respectively. Theorem 8 is a variant of Theorem 7 which is sometimes more convenient. 
Theorem 1. We have

$$
\begin{array}{ll}
\frac{x}{\log x}\left(1+\frac{1}{2 \log x}\right)<\pi(x) & \text { for } 59 \leqq x \\
\pi(x)<\frac{x}{\log x}\left(1+\frac{3}{2 \log x}\right) & \text { for } 1<x
\end{array}
$$

Theorem 2. We have

$$
\begin{array}{ll}
x /\left(\log x-\frac{1}{2}\right)<\pi(x) & \text { for } 67 \leqq x, \\
\pi(x)<x /\left(\log x-\frac{3}{2}\right) & \text { for } e^{3 / 2}<x
\end{array}
$$

(and hence for $4.48169 \leqq x$ ).

Corollary 1. We have

$$
\begin{aligned}
x / \log x<\pi(x) & \text { for } 17 \leqq x, \\
\pi(x)<1.25506 x / \log x & \text { for } 1<x .
\end{aligned}
$$

Corollary 2. For $1<x<113$ and for $113.6 \leqq x$

$$
\pi(x)<5 x /(4 \log x) .
$$

Corollary 3. We have

$$
\begin{aligned}
3 x /(5 \log x)<\pi(2 x)-\pi(x) & \text { for } 20 \frac{1}{2} \leqq x, \\
0<\pi(2 x)-\pi(x)<7 x /(5 \log x) & \text { for } 1<x .
\end{aligned}
$$

For the ranges of $x$ for which these corollaries do not follow directly from the theorem, they can be verified by reference to Lehmer's table of primes [10]. A similar remark applies to all corollaries of this section unless a proof is indicated.

The inequality (3.8) improves a result of Finsler [3]. The left side of (3.9) is just the classic result, conjectured by Bertrand (and known as Bertrand's Postulate) and proved in Tchebichef [14], that there is at least one prime between $x$ and $2 x$. The right side of (3.9) gives a result of Finsler [3], with Finsler's integral $n$ replaced by our real $x$. Finsler's elementary proofs are reproduced in Trost [15] on p. 58. The relation (3.12) below states a result of Rosser [11].

Theorem 3. We have

$$
\begin{array}{ll}
n\left(\log n+\log \log n-\frac{3}{2}\right)<p_{n} & \text { for } 2 \leqq n, \\
p_{n}<n\left(\log n+\log \log n-\frac{1}{2}\right) & \text { for } 20 \leqq n .
\end{array}
$$

Corollary. We have

$$
\begin{array}{cl}
n \log n<p_{n} & \text { for } 1 \leqq n, \\
p_{n}<n(\log n+\log \log n) & \text { for } 6 \leqq n .
\end{array}
$$


Theorem 4. We have

$$
\begin{aligned}
& x(1-1 /(2 \log x))<\vartheta(x) \\
& \vartheta(x)<x(1+1 /(2 \log x))
\end{aligned}
$$

for $563 \leqq x$, for $1<x$.

Corollary. We have

$$
x(1-1 / \log x)<\vartheta(x)
$$

for $41 \leqq x$.

Theorem 5. We have

$$
\begin{array}{cl}
\log \log x+B-1 /\left(2 \log ^{2} x\right)<\sum_{p \leqq x} 1 / p & \text { for } 1<x, \\
\sum_{p \leqq x} 1 / p<\log \log x+B+1 /\left(2 \log ^{2} x\right) & \text { for } 286 \leqq x .
\end{array}
$$

Corollary. We have

$$
\begin{array}{cl}
\log \log x<\sum_{p \leqq x} 1 / p & \text { for } 1<x, \\
\sum_{p \leqq x} 1 / p<\log \log x+B+1 / \log ^{2} x & \text { for } 1<x .
\end{array}
$$

Theorem 6. We have

$$
\begin{array}{ll}
\log x+E-1 /(2 \log x)<\sum_{p \leqq x}(\log p) / p & \text { for } 1<x \\
\sum_{p \leqq x}(\log p) / p<\log x+E+1 /(2 \log x) & \text { for } 319 \leqq x
\end{array}
$$

Corollary. We have

$$
\begin{array}{cl}
\sum_{p \leqq x}(\log p) / p<\log x+E+1 / \log x & \text { for } 32 \leqq x, \\
\sum_{p \leqq x}(\log p) / p<\log x & \text { for } 1<x .
\end{array}
$$

Theorem 7. We have

$$
\begin{array}{rlrl} 
& \frac{e^{-C}}{\log x}\left(1-\frac{1}{2 \log ^{2} x}\right)<\prod_{p \leqq x}\left(1-\frac{1}{p}\right) & \text { for } 285 \leqq x \\
\prod_{p \leqq x}\left(1-\frac{1}{p}\right)<\frac{e^{-C}}{\log x}\left(1+\frac{1}{2 \log ^{2} x}\right) & \text { for } \quad 1<x .
\end{array}
$$

Corollary. We have

$$
\frac{e^{-C}}{\log x}\left(1-\frac{1}{\log ^{2} x}\right)<\prod_{p \leqq x}\left(1-\frac{1}{p}\right) \quad \text { for } 1<x .
$$

Theorem 8. We have

$$
\begin{array}{ll}
e^{C}(\log x)\left(1-\frac{1}{2 \log ^{2} x}\right)<\prod_{p \leqq x} \frac{p}{p-1} & \text { for } 1<x, \\
\prod_{p \leqq x} \frac{p}{p-1}<e^{C}(\log x)\left(1+\frac{1}{2 \log ^{2} x}\right) & \text { for } 286 \leqq x .
\end{array}
$$

Corollary 1. We have

$$
\prod_{p \leqq x} \frac{p}{p-1}<e^{c}(\log x)\left(1+\frac{1}{\log ^{2} x}\right) \quad \text { for } 1<x
$$


Corollary 2. We have

$$
\prod_{p \leqq x} \frac{p}{p-1}<e^{c} \sum_{1 \leqq n \leqq x} \frac{1}{n} \quad \text { for } 1 \leqq x .
$$

Theorem 9. We have

$$
\vartheta(x)<1.01624 x \quad \text { for } 0<x .
$$

For a better bound for $\vartheta(x)$ when $x \leqq 10^{8}$, note Theorem 18 below.

Theorem 10. For $d \leqq x$, we have $c x<\vartheta(x)$ for each of the following pairs of values of $c$ and $d$ :

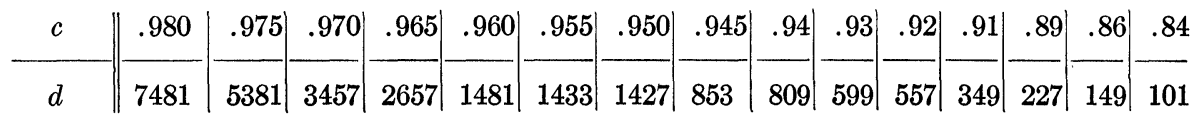

Theorem 11. Let

$$
R=\frac{515}{(\sqrt{546}-\sqrt{322})^{2}} \text { and } \varepsilon(x)=(\log x)^{1 / 2} \exp \{-\sqrt{(\log x) / R}\} .
$$

Then we have

$$
\begin{array}{ll}
\{1-\varepsilon(x)\} x<\vartheta(x) \leqq \psi(x) & \text { for } 2 \leqq x, \\
\vartheta(x) \leqq \psi(x)<\{1+\varepsilon(x)\} x & \text { for } 1 \leqq x .
\end{array}
$$

An approximate value for $R$ is

$$
R=17.51631 \text {. }
$$

Theorem 12. The quotient $\psi(x) / x$ takes its maximum at $x=113$, and

$$
\psi(x)<1.03883 x \quad \text { for } 0<x .
$$

Theorem 13. The quotient $\{\psi(x)-\vartheta(x)\} / x^{1 / 2}$ takes its maximum at $x=361$, and

$$
\psi(x)-\vartheta(x)<1.42620 x^{1 / 2} \quad \text { for } 0<x .
$$

Theorem 14. We have

$$
\begin{aligned}
0.98 x^{1 / 2}<\psi(x)-\vartheta(x) & \text { for } 121 \leqq x, \\
\psi(x)-\vartheta(x)<\vartheta\left(x^{1 / 2}\right)+3 x^{1 / 3} & \text { for } 0<x .
\end{aligned}
$$

Corollary. We have

$$
\psi(x)-\vartheta(x)<1.02 x^{1 / 2}+3 x^{1 / 3} \quad \text { for } 0<x .
$$

Proof. Use Theorem 9.

Theorem 15 . For $2 \leqq n$

$$
1+1 /(n-1) \leqq n / \phi(n) ;
$$


also for $3 \leqq n$

$$
n / \phi(n)<e^{C} \log \log n+5 /(2 \log \log n)
$$

except when

in which case

$$
n=223092870=2 \cdot 3 \cdot 5 \cdot 7 \cdot 11 \cdot 13 \cdot 17 \cdot 19 \cdot 23
$$

$$
n / \phi(n)<e^{c} \log \log n+2.50637 / \log \log n .
$$

In (3.40), equality is attained whenever $n$ is a prime. Thus, by taking $n$ to be a large prime, we can make $n / \phi(n)$ arbitrarily close to unity. It is shown in Landau [7], pp. 217-219, vol. 1, that for each positive $\varepsilon$ there are an infinity of $n$ 's for which

$$
\left(e^{c}-\varepsilon\right) \log \log n<n / \phi(n) .
$$

We do not know if there are an infinity of $n$ 's for which

$$
e^{c} \log \log n \leqq n / \phi(n)
$$

\section{Special approximations for limited ranges}

Theorem 16. We have

$$
\begin{gathered}
\operatorname{li}(x)-\operatorname{li}\left(x^{1 / 2}\right)<\pi(x) \\
\pi(x)<\operatorname{li}(x)
\end{gathered}
$$$$
\text { for } 11 \leqq x \leqq 10^{8}
$$$$
\text { for } 2 \leqq x \leqq 10^{8} \text {. }
$$

Theorem 17. We have

$$
x-\{\operatorname{li}(x)-\pi(x)\} \log x<\vartheta(x) \quad \text { for } e^{3} \leqq x \leqq 10^{8},
$$

(4.4) $\vartheta(x)<x-2 x^{1 / 2}+\left\{\pi(x)-\operatorname{li}(x)+\operatorname{li}\left(x^{1 / 2}\right)\right\} \log x$ for $e^{4} \leqq x \leqq 10^{8}$.

By use of Theorem 17 and the Rand table of primes [1], one can get quite sharp estimates of $\vartheta(x)$ for $e^{4} \leqq x \leqq 10^{8}$. However, it is usually adequate to use the more convenient but less precise results below.

THEOREM 18. For $0<x \leqq 10^{8}$

$$
x-2.05282 x^{1 / 2}<\vartheta(x)<x .
$$

Theorem 19. For $0<x \leqq 1420.9$ and for $1423 \leqq x \leqq 10^{8}$

$$
x-2 x^{1 / 2}<\vartheta(x) \text {. }
$$

The coefficient in (4.5) corrects a transposition of digits in Theorem 6 of Rosser [12].

Theorem 20. For $1<x \leqq 10^{8}$

$$
\log \log x+B<\sum_{p \leqq x} 1 / p<\log \log x+B+2 /\left(x^{1 / 2} \log x\right) .
$$

TheOREM 21. For $0<x \leqq 10^{8}$

(4.8) $\log x+E<\sum_{p \leqq x}(\log p) / p<\log x+E+2.06123 / x^{1 / 2}$. 
Theorem 22. For $0<x<113$ and for $113.8 \leqq x \leqq 10^{8}$

$$
\sum_{p \leqq x}(\log p) / p<\log x+E+2 / x^{1 / 2} .
$$

Theorem 23. For $0<x \leqq 10^{8}$

$$
e^{C} \log x<\prod_{p \leqq x} p /(p-1)<e^{C} \log x+2 e^{C} / x^{1 / 2} .
$$

Theorem 24. We have

$$
\begin{array}{cc}
x^{1 / 2}<\psi(x)-\vartheta(x) & \text { for } 121 \leqq x \leqq 10^{16}, \\
\psi(x)-\vartheta(x)<x^{1 / 2}+3 x^{1 / 3} & \text { for } \quad 0<x \leqq 10^{16} .
\end{array}
$$

One immediately wonders if the results of Theorems 20-23 could be valid for all $x$. It is known that each of (4.1), (4.2), and (4.5) fails infinitely often for large $x$, and indeed each side of (4.5) fails infinitely often (see Theorems 35 and 34 on pp. 103 and 100 of Ingham [6]). Perhaps one can extend these results to show that each of (4.7) through (4.10) fails for large $x$; we have not investigated the matter.

Theorem 18 gives sharp bounds for $\vartheta(x)$ for $0<x \leqq 10^{8}$. For larger values of $x$, sharp bounds for $\vartheta(x)$ can be obtained by use of Theorem 14 and its corollary provided sharp bounds for $\psi(x)$ are known. For $10^{8} \leqq x \leqq$ $e^{5000}$, sharp bounds for $\psi(x)$ can be obtained from our 'Table I, in which we tabulate against $b$ values of $\varepsilon$ such that for $e^{b} \leqq x$

$$
(1-\varepsilon) x<\psi(x)<(1+\varepsilon) x .
$$

The values of $m$ listed pertain to the computations by which Table I was established, all of which will be explained later.

Finally, Theorem 11 can be used to get close approximations to both $\psi(x)$ and $\vartheta(x)$ for large $x$ beyond the range of existing tables. Although Theorem 11 is valid for small values of $x$ as well as large, for $x$ below about $e^{3000}$ it gives poorer estimates for $\vartheta(x)$ than can be obtained from Theorems 4, 9, 10, 18, and 19. From $e^{18.4}$ to $e^{4800}$, Theorem 11 gives poorer estimates than can be obtained from Table I with the help of Theorem 14.

We can use our sharp estimates for $\vartheta(x)$ to get sharp estimates for other functions depending on primes. Using the Stieltjes integral, we can write

$$
\sum_{p \leqq x} f(p)=\int_{2-}^{x} \frac{f(y)}{\log y} d \vartheta(y) .
$$

An integration by parts gives

$$
\sum_{p \leqq x} f(p)=\frac{f(x) \vartheta(x)}{\log x}-\int_{2}^{x} \vartheta(y) \frac{d}{d y}\left(\frac{f(y)}{\log y}\right) d y .
$$

Alternatively, one can derive (4.13) by use of Theorem A on p. 18 of Ingham 
[6]. From (4.13), as in the derivation of (2.26), we get

$$
\begin{aligned}
\sum_{p \leqq x} f(p)= & \int_{2}^{x} \frac{f(y) d y}{\log y}+\frac{2 f(2)}{\log 2} \\
& +\frac{f(x)\{\vartheta(x)-x\}}{\log x}-\int_{2}^{x}\{\vartheta(y)-y\} \frac{d}{d y}\left(\frac{f(y)}{\log y}\right) d y .
\end{aligned}
$$

For suitable $f$, we can write (4.14) as

$$
\begin{aligned}
\sum_{p \leqq x} f(p)=\int_{2}^{x} & \frac{f(y) d y}{\log y}+L_{f} \\
& \quad+\frac{f(x)\{\vartheta(x)-x\}}{\log x}+\int_{x}^{\infty}\{\vartheta(y)-y\} \frac{d}{d y}\left(\frac{f(y)}{\log y}\right) d y
\end{aligned}
$$

where $L_{f}$ is the constant given by

$$
L_{f}=\frac{2 f(2)}{\log 2}-\int_{2}^{\infty}\{\vartheta(y)-y\} \frac{d}{d y}\left(\frac{f(y)}{\log y}\right) d y .
$$

From (4.13) we get

$$
\begin{aligned}
\pi(x) & =\frac{\vartheta(x)}{\log x}+\int_{2}^{x} \frac{\vartheta(y) d y}{y \log ^{2} y} \\
\sum_{p \leqq x} \frac{1}{p} & =\frac{\vartheta(x)}{x \log x}+\int_{2}^{x} \frac{\vartheta(y)(1+\log y)}{y^{2} \log ^{2} y} d y \\
\sum_{p \leqq x} \frac{\log p}{p} & =\frac{\vartheta(x)}{x}+\int_{2}^{x} \frac{\vartheta(y) d y}{y^{2}} .
\end{aligned}
$$

From (4.15) we get

$$
\begin{aligned}
& \sum_{p \leqq x} \frac{1}{p}=\log \log x+B+\frac{\vartheta(x)-x}{x \log x} \\
&-\int_{x}^{\infty} \frac{\{\vartheta(y)-y\}(1+\log y)}{y^{2} \log ^{2} y} d y
\end{aligned}
$$

$$
\sum_{p \leqq x} \frac{\log p}{p}=\log x+E+\frac{\vartheta(x)-x}{x}-\int_{x}^{\infty} \frac{\vartheta(y)-y}{y^{2}} d y
$$

To prove (4.20), it suffices to show that in this case $L_{f}-\log \log 2=B$; to show this, we let $x \rightarrow \infty$ in (4.15) and use Theorem 11 and the definition of $B$. The proof of $(4.21)$ is similar.

\section{Tabular and computational results, and proofs derived therefrom}

The well-known table of primes, Lehmer [10], lists all primes less than $10^{7}$ in such a fashion that one can easily obtain the corresponding value of $\pi(x)+1$; since Lehmer takes unity to be the first prime, his count of primes 
differs by unity from ours. Just recently the Rand Corporation has prepared a list of primes, published on microcards in Baker and Gruenberger [1], giving all primes up to slightly beyond $10^{8}$. Within the large range of these tables, one can read off exact values of $\pi(x)$ and $p_{n}$. Their count agrees with Lehmer's rather than ours.

By use of Lehmer's Table, Rosser verified Theorem 16 and the right half of (4.5) for $x \leqq 10^{6}$. An account of his methods is given in Rosser [12]. By the same methods, these results were extended to $10^{7}$ in the first major computation cited in Section 1.

We turn next to the calculations required to establish (2.10) through (2.14). The values for $c(1)$ and $1 / c(1)$ given there were computed from (2.9) and the known value of $C$. The values for $B$ and $c(2)$ given in (2.10) and (2.14) have been taken from pp. 43 and 44 of Rosser [11]. At the end of the footnote on p. 43 of this reference, there is given a twenty-four-decimal value of $C-B$; this value corrects the slightly erroneous value of $B-C$ given in Table I of Gram [5]. Incidentally, Gram reproduces Merrifield's incorrect value of $\sum p^{-3}$ in his Table I; a correct value is given on p. 249 of Davis [2].

To determine the remaining quantity, $E$, we use the relation

$$
-\zeta^{\prime}(s) / \zeta(s)=\sum_{p}(\log p) /\left(p^{s}-1\right)=\sum_{p} \sum_{r=1}^{\infty}(\log p) / p^{r s}
$$

which is given near the bottom of p. 17 of Ingham [6]. From this we obtain for $n>1$

$$
\sum_{p}(\log p) / p^{n}=-\sum_{m=1}^{\infty} \mu(m) \zeta^{\prime}(m n) / \zeta(m n)
$$

Substituting this into $(2.8)$ gives

$$
E=-C-\sum_{m=2}^{\infty} \mu(m) \zeta^{\prime}(m) / \zeta(m) .
$$

We first computed $\zeta^{\prime}(n)$ by using an electronic computer to sum the first 500 terms of the series

$$
\zeta^{\prime}(n)=-\sum_{m=2}^{\infty}(\log m) / m^{n}
$$

the remainder of the series was computed by the Euler-Maclaurin sum formula. As a check, this was repeated with the first 1000 terms. Using the values of $\zeta(n)$ given on p. 244 of Davis [2], values of

$$
-\zeta^{\prime}(n) / \zeta(n) \text { and } \sum_{p}(\log p) / p^{n}
$$

were computed for $2 \leqq n \leqq 56$ by using (5.1). These, together with the values of $-\zeta^{\prime}(n)$ are listed for $2 \leqq n \leqq 29$ in Table IV. In this table the values given for $-\zeta^{\prime}(n)$ are in error by less than $10^{-17}$. Division by $\zeta(n)$ could cause slightly greater errors in $-\zeta^{\prime}(n) / \zeta(n)$. As we used seventeendecimal values in (5.1), the errors in $\sum_{p} p^{-n} \log p$ could be a bit greater. For $n>29$, the three functions tabulated do not differ in the first seventeen decimals, and each is given to better than seventeen decimals by

$$
(\log 2) / 2^{n}+(\log 3) / 3^{n} \text {. }
$$


Using (2.8), we got the approximation

$$
E=-1.33258227573322087
$$

which we checked by (5.2).

Incidentally, our values of $-\zeta^{\prime}(n) / \zeta(n)$ check the seven-decimal values given in Walther [16].

With values for $B, E$, and $e^{-C}$ now established, other computations could be undertaken. The second major computation cited in Section 1 was the tabulation by Rosser and Walker of many functions of primes for $x \leqq 16,000$. This verified Theorems 1-10 and their corollaries in this range, with the exception of Theorem 3 , which was established for $p_{n} \leqq 16,000$. This computation also verified Theorems $18-23$ for $x \leqq 16,000$.

The third major computation cited in Section 1 was the tabulation by Appel and Rosser of many functions of primes for $x \leqq 10^{8}$. This established Theorems 16 and $18-23$ for $313 \leqq x \leqq 10^{8}$, which completed their verification. A discussion of this computation, together with a partial tabulation and many more details can be found in a report written by Appel and Rosser [19].

Theorem 17 is an immediate consequence of Theorem 16 as a result of Lemmas 5 and 6 of Rosser [12]. Thus Theorems 16 through 23 are established.

In Table II, at the end of this paper, we have listed values of $\vartheta(x)$ and other functions selected from the Rosser-Walker tabulation. If an isolated value is desired in the range $x \leqq 16,000$, it can be readily computed by working from the nearest entry in Table II; it can be checked by working from the entry on the other side. If numerous random values are desired, it is probably easier to generate an entire table on one of the modern very fast computers, using the entries in Table II to check key values. Other values for comparison are available in the report by Appel and Rosser [19].

For more limited ranges, one can derive values of some functions from tables already in the literature. Thus in Glaisher [4] is given a seven-decimal table of

$$
f(x)=\prod_{p \leqq x}(1-1 / p)
$$

and its common logarithm for $2 \leqq x \leqq 10,000$. By comparison with the Rosser-Walker tabulation, we verified that the Glaisher table is quite reliable. Round-off errors are common in the function values, but we found only four cases where the listed function value is in error by more than one unit in the last place. The correct values there are as follows:

$$
\begin{aligned}
& f(4271)=0.0670040, \quad f(9397)=0.0613076, \\
& f(8609)=0.0619246, \quad f(9883)=0.0609460 .
\end{aligned}
$$

In all these cases, the logarithmic values given were accurate to within round- 
off errors. The last column of our Table II gives selected values of $1 / f(x)$ to ten decimals.

In Gram [5] is given an eight-decimal table of $\psi(x)$ for $x \leqq 2000$. The eighth decimal is quite unreliable, but no entry is in error by as much as $10^{-7}$. From this, one can readily compute values of $\vartheta(x)$ for $x \leqq 2000$ by using our Table III, which gives the values of $\psi(x)-\vartheta(x)$ for $x<50,653$. The arguments $x$ are those prime powers $p^{r}$ having $r \geqq 2$, so that the tabulated function is constant between entries. By means of Table III one can also compute $\psi(x)$ from the values of $\vartheta(x)$ given in Table II up to $x=16,000$.

We now turn to Theorems 12-14, 24. We first take note of the following result from pp. 90-91 of Landau [7], vol. 1, proved by adapting the elementary derivation of Tchebichef [14].

Theorem 25. For $1 \leqq x$

$$
\vartheta(x) \leqq \psi(x)<1.2 a x+(3 \log x+5)(\log x+1),
$$

where a denotes the constant

$$
\frac{7}{15} \log 2+\frac{3}{10} \log 3+\frac{1}{6} \log 5=0.92129 \cdots,
$$

according to the definition on p. 88 of Landau [7], vol. 1.

Using (5.3) with Theorem 18 gives the following weakened form of Theorem 9:

$$
\vartheta(x)<1.11 x \quad \text { for } 0<x .
$$

Using values of $\vartheta\left(x^{1 / 2}\right)$ from the Rosser-Walker computation, we verified (3.38) for $x<50,653$ by using Table III. For $50,653 \leqq x<10^{24}$, one can verify (3.38) by (2.24) and the right side of (4.5); to do this, we proceed by cases such as $2^{M} \leqq x<2^{N}$ where $M$ and $N$ are conveniently chosen integers. Finally, for $10^{24} \leqq x,(3.38)$ holds by (2.24) and (5.4). Thus (3.38) has been completely established. From it, by (5.4) one can infer the following weakened form of $(3.39)$ :

$$
\psi(x)-\vartheta(x)<1.11 x^{1 / 2}+3 x^{1 / 3} \quad \text { for } 0<x .
$$

As (3.38) and the right side of (4.5) imply (4.12), we can complete the proof of Theorem 24 by establishing (4.11). This is readily done for $121 \leqq$ $x<50,653$ by means of Table III. By using Theorems 18 and 19 with (2.24), we can finish the proof of (4.11) and hence of Theorem 24.

By comparison with Gram's Table [5] of $\psi(x)$, Theorem 12 was verified for $x \leqq 2000$, and it was ascertained that in this range the maximum value of $\psi(x) / x$ lies between 1.03882 and 1.03883. Thus one can complete the proof of Theorem 12 by proving that $\psi(x) \leqq 1.03882 x$ for $2000 \leqq x$. This follows for $x<50,653$ by Theorem 18 and Table III. For $50,653 \leqq x \leqq 10^{8}$ it follows by Theorem 18 and (4.12). It will follow for all greater $x$ by Table 
I as soon as we have justified the values in this table, which we will do in the next section.

We can verify Theorem 13 in the range $0<x<50,653$ by reference to Table III. It then suffices to verify $\psi(x)-\vartheta(x) \leqq 1.42619 x^{1 / 2}$ for $50,653 \leqq$ $x$. For $10^{6} \leqq x$, we infer this by (5.5). For $x<10^{6}$ we use $(2.25)$; the facts that $\psi(y)=0$ for $y<2$ and that $\psi$ is monotone let us conclude

$$
\begin{gathered}
\psi(x)-\vartheta(x)=\psi\left(x^{1 / 2}\right)+\psi\left(x^{1 / 3}\right)+\psi\left(x^{1 / 5}\right)-\psi\left(x^{1 / 6}\right) \\
+\psi\left(x^{1 / 7}\right)-\psi\left(x^{1 / 10}\right)+\psi\left(x^{1 / 11}\right)+\psi\left(x^{1 / 13}\right) \\
-\psi\left(x^{1 / 14}\right)-\psi\left(x^{1 / 15}\right)+\psi\left(x^{1 / 17}\right)+\psi\left(x^{1 / 19}\right) \\
\leqq \psi\left(x^{1 / 2}\right)+\psi\left(x^{1 / 3}\right)+\psi\left(x^{1 / 5}\right)+\psi\left(x^{1 / 13}\right) \\
<1.04\left(x^{1 / 2}+x^{1 / 3}+x^{1 / 5}+x^{1 / 13}\right)
\end{gathered}
$$

since (3.35) holds for $x \leqq 10^{8}$. This suffices to complete the proof.

At this point, therefore, we have established (2.10) through (2.14), Theorems 16 through 24, Theorem 12 except for $10^{8}<x$, Theorem 13 , and (3.38) of Theorem 14. We have also verified Theorems 1 through 10 and their corollaries for $x \leqq 16,000$ or for $p_{n} \leqq 16,000$.

\section{Sharpening of some results of Rosser, with application to several proofs}

In the preceding section, we carried our proofs as far as is practicable without appealing to very deep results. From here on, we shall be mainly concerned with invoking certain deep results to validate Table I and complete the proofs of the results stated in Section 3. Space does not permit us to give proofs in full, so that we shall assume that the reader is quite familiar with Ingham [6] and Rosser [12], from which we shall use notation and results with a minimum of reference.

The most significant sharpening of results from Rosser [12] arises from the fact that it is now known that the first 25000 zeros of the zeta function have real part equal to $\frac{1}{2}$, as shown in Lehmer [8] and Lehmer [9]. This enables us to replace the $A$ on p. 223 of Rosser [12] by $A=e^{9.99}$. We do not now have $N(A)=F(A)$, which will make a slight change in a key formula, as we note below.

Observing that

$322+546 \cos \phi+329 \cos 2 \phi+130 \cos 3 \phi+25 \cos 4 \phi$

$$
=2(1+\cos \phi)^{2}(3+10 \cos \phi)^{2} \geqq 0,
$$

we can modify the proof of Theorem 20 of Rosser [12] to get a proof of

Theorem 26. For $A \leqq \gamma$, we have $\beta<1-1 /(R \log \gamma)$.

The $R$ here is that defined in Theorem 11. In other places, as here, it replaces the number 17.72 appearing in Rosser [12]. Thus, we are conforming 
with the notation of Rosser [12] when we temporarily abrogate the usual denotation of $\phi(n)$ and define

$$
\phi(\gamma)=\phi(m, x, \gamma)=\gamma^{-m-1} e^{-(\log x) /(R \log \gamma)} .
$$

However, for the purposes of the next theorem we consider (6.1) as defining $\phi(\gamma)$ for arbitrary positive $R$.

Theorem 27. If $\phi(\gamma)$ is defined as in (6.1) with $m$ and $R$ positive numbers, if $2 \leqq K$, and if $0 \leqq \log x \leqq(m+1) R \log ^{2} K$, then

$$
\sum_{K<\gamma} \phi(\gamma)<2 R(K) \phi(K)+Q \int_{K}^{\infty} \phi(y) \log \frac{y}{2 \pi} d y
$$

where

$$
Q=\frac{1}{2 \pi}+\frac{0.137 \log K+0.443}{K \log K \log (K / 2 \pi)} .
$$

Proof. Proceed as in the proof of Lemma 18 of Rosser [12].

Corollary. If in (6.1) we take $R$ to be the $R$ of Theorem 11, and if $A \leqq K$, $0<m$, and

$$
0 \leqq \log x<1748(m+1)
$$

then

$$
\sum_{K<\gamma} \phi(\gamma)<2 R(K)_{\phi}(K)+0.1592 \int_{K}^{\infty} \phi(y) \log \frac{y}{2 \pi} d y
$$

Proof. As we are here using the $R$ of Theorem 11, (6.4) verifies the final hypothesis of Theorem 27. In (6.5), the coefficient in front of the integral is got by taking $K=e^{9.99}$ in (6.3), which is permissible since $e^{9.99}=A \leqq K$.

In Rosser [12], in the situation corresponding to taking $K=A$ in the corollary, the coefficient 2 did not appear in the first term on the right of (6.5). This is because $N(A)=F(A)$ in that paper. Except for that, we now proceed as in the proofs of Lemma 19 and Theorem 21 of Rosser [12] to derive

THEOREM 28. If $m$ is a positive integer,

$$
\begin{aligned}
& \sum_{\rho} \frac{1}{\left|\gamma^{m+1}\right|} \leqq k_{m}, \quad \log a<\frac{1748 m^{2}}{m+0.123}, \\
& \delta \geqq 2\left\{\frac{k_{m}}{a^{1 / 2}}+\frac{0.0003647 m^{2}+1.298 m+0.1592}{\left(1-\frac{m+0.123}{1748 m^{2}} \log a\right) m^{2} A^{m} a^{1 / 175}}\right\}^{1 /(m+1)}, \\
& \varepsilon=\frac{\delta}{2}\left\{\left(\frac{(1+\delta)^{m+1}+1}{2}\right)^{m}+m\right\} \text {, }
\end{aligned}
$$

and $1+m \delta a<a$, then for $a \leqq x$

$$
x(1-\varepsilon)-1.84<\psi(x)<x(1+\varepsilon)-\frac{1}{2} \log \left(1-x^{-2}\right) .
$$


By taking $a$ successively equal to $e^{b}$ for the various values of $b$ listed in Table I, and using with these values the listed values of $m$, the values of $\varepsilon$ listed in Table I were derived from Theorem 28. In particular, the value for $b=5000$ was listed just as it was given by Theorem 28 despite the fact that Theorem 28 gives a smaller $\varepsilon$ for $b=4900$, as listed in Table I. The bounds used for $k_{1}, k_{2}$, and $k_{3}$ are those given in Lemma 17 of Rosser [12]. Bounds for larger $m$ were obtained by the trivial inequality

$$
14 \sum\left|\gamma^{-m-2}\right|<\sum\left|\gamma^{-m-1}\right| \text {. }
$$

Now that we have justified Table I, we use it to complete the proof of Theorem 12, as noted above.

Turning to Theorem 9 , we verify it for $x \leqq 10^{8}$ by Theorem 18 . For $10^{8} \leqq x \leqq 10^{16}$, we have $\vartheta(x)<\psi(x)-x^{1 / 2}$ by Theorem 24 , and so verify Theorem 9 in this range by Table I. Above $10^{16}$, we use Table I with the trivial inequality $\vartheta(x)<\psi(x)$.

We complete the verification of Theorem 10 for $16,000 \leqq x \leqq 10^{8}$ by Theorem 19. Above this, we use (5.5) with Table I.

As far as it furnishes bounds on $\vartheta(x)$, we verify Theorem 11 for $x \leqq 101$ by comparison with values of $\vartheta(x)$ taken from the Rosser-Walker tabulation. Now with $\varepsilon$ defined as in Theorem 11 , we have $\varepsilon \geqq 0.625$ for $2 \leqq x \leqq e^{9 R}$. Thus we can complete the verification of Theorem 11 in this range by Theorems 10 and 12. This puts us in the range of Table I. From here to $e^{4800}$ we can proceed by using Table I with (5.5). We now complete the proof of Theorem 11 as in the proof of Theorem 22 of Rosser [12], noting that by (5.5) the difference between $\vartheta(x)$ and $\psi(x)$ is so small as to be more than allowed for by the fact that various quantities do not actually attain the upper bounds by which they are replaced in the proof.

We verify (3.37) for $x \leqq 10^{16}$ by Theorem 24 . For greater $x$, it follows by (2.24) and Theorem 10. Thus we have completed the proof of Theorem 14.

For some of our later proofs we will need results that are sharper in certain ranges than Theorem 4 . We now state and prove several such results.

Theorem 29. For $1451 \leqq x \leqq e^{375}$,

$$
x\left(1-\frac{0.31}{\log x}\right)<\vartheta(x)<x\left(1+\frac{0.31}{\log x}\right) .
$$

Theorem 30. For $809 \leqq x \leqq e^{575}$,

$$
x\left(1-\frac{0.40}{\log x}\right)<\vartheta(x)<x\left(1+\frac{0.40}{\log x}\right) .
$$

Theorem 31. For $569 \leqq x$,

$$
x\left(1-\frac{0.47}{\log x}\right)<\vartheta(x)<x\left(1+\frac{0.47}{\log x}\right) .
$$

For $x \leqq 16,000$, these are established by means of the Rosser-Walker tabu- 
lation. For $16,000 \leqq x \leqq 10^{8}$, these are established by Theorems 18 and 19 . For $10^{8} \leqq x \leqq 10^{16}$, we use Theorem 24 and Table I. For $10^{16} \leqq x \leqq e^{5000}$, we use the corollary of Theorem 14 and Table I. Finally, above $e^{5000}$ we use Theorem 11.

From these, Theorem 4 is an easy consequence.

\section{Proof of Theorems 1 through 3}

We start with five lemmas. As their proofs are similar, we state all five lemmas first before giving the proofs. We first make the definition

$$
\begin{aligned}
J(x, a)=\pi(1451)-\frac{\vartheta(1451)}{\log 1451}+\frac{x}{\log x}(1 & \left.+\frac{a}{\log x}\right) \\
& +\int_{1451}^{x}\left(1+\frac{a}{\log y}\right) \frac{d y}{\log ^{2} y} .
\end{aligned}
$$

LEMma 1. For $e^{8} \leqq x$,

$$
\operatorname{li}(x)<\frac{x}{\log x}\left(1+\frac{3}{2 \log x}\right) .
$$

LEMMA 2. For $10^{8} \leqq x$ and $a=0.31$,

$$
J(x, a)<\frac{x}{\log x}\left(1+\frac{3}{2 \log x}\right) .
$$

LEMmA 3. For $e^{100} \leqq x$ and $a=0.47$, the inequality (7.3) is valid.

LEMma 4. For $e^{5} \leqq x$,

$$
x /\left(\log x-\frac{1}{2}\right)<\operatorname{li}(x)-\operatorname{li}\left(x^{1 / 2}\right) .
$$

Lemma 5. For $10^{8} \leqq x$ and $a=-0.47$,

$$
x /\left(\log x-\frac{1}{2}\right)<J(x, a) .
$$

For each of these lemmas, the proof is in two parts. First, one verifies that in the stated range of $x$, the derivative of the left side is less than that of the right side. Second, one verifies that at the lower limit of $x$ the left side is less than the right side. To perform the needed calculations, one can use the reduction formula

$$
\int_{b}^{x} \frac{a d y}{\log ^{a+1} y}=\frac{b}{\log ^{a} b}-\frac{x}{\log ^{a} x}+\int_{b}^{x} \frac{d y}{\log ^{a} y}
$$

to express the various integrals in terms of $\mathrm{li}(x)$ and elementary functions. For $x \leqq e^{15}$, one can get numerical values of $\operatorname{li}(x)$ from the tables of $\operatorname{Ei}(y)$ given in [18]. Outside this range, one can appeal to the following result.

Theorem 32. If $m$ is a positive integer and $m \leqq y \leqq m+1$, then

$$
\frac{2}{3}\left(\frac{2 \pi}{m}\right)^{1 / 2}-\frac{1.06}{m}+e^{y} \sum_{j=1}^{m-1} \frac{(j-1) !}{y^{j}}
$$


and

$$
\frac{2}{3}\left(\frac{2 \pi}{m+1}\right)^{1 / 2}+\frac{1.06}{m+1}+e^{y} \sum_{j=1}^{m} \frac{(j-1) !}{y^{j}}
$$

are lower and upper bounds respectively for $\operatorname{Ei}(y)$.

This is a consequence of equations (58), (68), and (69), and of Lemma 3 and Theorem 7 of Rosser [13].

We note the value $\pi(1451)=230$ and the approximation $\vartheta(1451)=1396.4$ taken from the Rosser-Walker tabulation.

We now turn to (3.2), which has already been established for $x \leqq 16,000$ by the Rosser-Walker tabulation. By Lemma 1 and Theorem 16, we verify (3.2) up to $x=10^{8}$. We have by (4.17) that

$$
\pi(x)=\pi(1451)-\frac{\vartheta(1451)}{\log 1451}+\frac{\vartheta(x)}{\log x}+\int_{1451}^{x} \frac{\vartheta(y) d y}{y \log ^{2} y} .
$$

By Theorem 29, we can conclude $\pi(x)<J(x, 0.31)$ if $1451 \leqq x \leqq e^{375}$. So by Lemma 2 we can infer (3.2) for $10^{8} \leqq x \leqq e^{375}$. In a similar way we combine Lemma 3 and Theorem 31 to complete the proof of (3.2).

In a similar way, we combine Lemma 4 and Theorem 16 to verify (3.3) for $x \leqq 10^{8}$, and complete the verification by combining Lemma 5 and Theorem 31 .

We get (3.1) from (3.3), and (3.4) from (3.2), by applying the inequality

$$
\frac{x}{\log x}\left(1+\frac{a}{\log x}\right)<\frac{x}{\log x-a}
$$

for $e^{a}<x$

in the two cases $a=\frac{1}{2}$ and $a=\frac{3}{2}$.

As preliminaries for the proof of Theorem 3 we undertake the proof of (3.12) and

$$
p_{n}<n(\log n+2 \log \log n) \quad \text { for } \quad 4 \leqq n .
$$

These were proved in Rosser [11], but can be derived so readily from the strong results now available that it seems worthwhile to indicate new proofs by this method. For instance, suppose if possible that $p_{n} \leqq n \log n$. Then

So by (3.2), we have

$$
n \leqq \pi(n \log n) \text {. }
$$

$$
n<\frac{n \log n}{\log n+\log \log n}\left(1+\frac{1.5}{\log n+\log \log n}\right),
$$

a result which certainly fails if $e^{5} \leqq n$. So (3.12) holds for $e^{5} \leqq n$, and a trivial computation verifies it for smaller $n$. The proof of (7.9) is analogous.

From these, we can now infer Lemmas 9 and 10 of Rosser [12], using the proofs given there. Using Lemma 9 of Rosser [12] with Theorem 18 gives

$$
n \log n+n \log \log n-n-\operatorname{li}(n)<p_{n}
$$


for $5 \leqq n \leqq \pi\left(10^{8}\right)$. Then (3.10) follows for $e^{4} \leqq n \leqq \pi\left(10^{8}\right)$ by Lemma 7 of Rosser [12]. Now use Lemma 9 of Rosser [12] with Theorem 30, and infer

$$
n \log n+n \log \log n-n-\operatorname{li}(n)<p_{n}\left(1+\frac{0.40}{\log p_{n}}\right)
$$

for $140 \leqq n \leqq e^{568}$. As li $(n)<0.1 n$ for $\pi\left(10^{8}\right) \leqq n$, by Lemma 7 of Rosser [12], and

$$
p_{n} /\left(\log p_{n}\right)<n
$$

by (3.5), we infer (3.10) for $n \leqq e^{568}$. We use Theorem 31 in a similar manner to complete the proof of (3.10).

We next prove (3.11). We note first of all that it has been verified for $n \leqq 1862$ by the Rosser-Walker tabulation. Now let $1862 \leqq n$, and suppose that (3.11) has been verified for all integers less than $n$. Then the hypothesis of Lemma 10 of Rosser [12] is verified, and we conclude that

$$
\vartheta\left(p_{n}\right)<n \log n+n \log \log n-n+\frac{n \log \log n}{\log n} .
$$

By Theorem 19 , if $n \leqq \pi\left(10^{8}\right)$, then

$$
p_{n}-2\left(p_{n}\right)^{1 / 2}<n \log n+n \log \log n-n+\frac{n \log \log n}{\log n} .
$$

By (7.9),

$$
2\left(p_{n}\right)^{1 / 2}<2(n \log n+2 n \log \log n)^{1 / 2}<0.2 n,
$$

so that (3.11) is verified. Now let $\pi\left(10^{8}\right) \leqq n \leqq e^{369}$. Then (7.11) and Theorem 29 give

$$
p_{n}\left(1-\frac{0.31}{\log p_{n}}\right)<n \log n+n \log \log n-n+\frac{n \log \log n}{\log n} .
$$

Using (7.10), we again infer (3.11). In a similar way, we can use Theorem 31 to verify (3.11) for $e^{369} \leqq n$.

\section{Proof of Theorems 5 through 8}

We require several lemmas.

Lemma 6. For $1 \leqq x$ and $A \leqq K$,

$$
K(1, x)<\frac{0.0463}{x^{1 / 2}}+\frac{2 R(K)}{K^{2}}+0.1592 \frac{1+\log (K / 2 \pi)}{K}+e^{-9.61} x^{-1 /(R \log K)} .
$$

Proof. Taking $m=1=x$ in (6.5) gives

$$
\sum_{K<\gamma} \frac{1}{\gamma^{2}}<\frac{2 R(K)}{K^{2}}+0.1592 \frac{1+\log (K / 2 \pi)}{K} .
$$


Taking $K=A$ in this gives

$$
\sum_{A<\gamma} \frac{1}{\gamma^{2}}<e^{-9.61}
$$

By Lemmas 16 and 17 of Rosser [12]

$$
\begin{aligned}
K(1, x) & <\frac{0.0463}{x^{1 / 2}}+\sum_{A<\gamma \leqq K} \phi(\gamma)+\sum_{K<\gamma} \phi(\gamma) \\
& <\frac{0.0463}{x^{1 / 2}}+\sum_{A<\gamma \leqq K} \frac{x^{-1 /(R \log K)}}{\gamma^{2}}+\sum_{K<\gamma} \frac{1}{\gamma^{2}} .
\end{aligned}
$$

Our lemma now follows by use of (8.1) and (8.2).

We next state two lemmas whose proofs are so similar that we give only the more difficult one, namely that for Lemma 8.

LEMMA 7. For $1<x$,

$$
\left|\int_{x}^{\infty} \frac{y-\psi(y)}{y^{2}} d y\right|<K(1, x)+\frac{1.84}{x}+\frac{0.31}{x^{3}} .
$$

LemMa 8. For $1<x$,

$$
\left|\int_{x}^{\infty} \frac{(y-\psi(y))(1+\log y)}{y^{2} \log ^{2} y} d y\right|<\frac{2+\log x}{\log ^{2} x}\left(K(1, x)+\frac{1.84}{x}+\frac{0.31}{x^{3}}\right) \text {. }
$$

Proof. We have

$$
\int_{x}^{\infty} \frac{y^{\rho-2}(1+\log y)}{\log ^{2} y} d y=\frac{-x^{\rho-1}}{(\rho-1) \log x}-\frac{\rho}{(\rho-1)^{2}}\left\{\frac{x^{\rho-1}}{\log ^{2} x}-2 \int_{x}^{\infty} \frac{y^{\rho-2}}{\log ^{3} y} d y\right\} .
$$

Now

So

$$
\begin{aligned}
\left|\frac{x^{\rho-1}}{\log ^{2} x}-2 \int_{x}^{\infty} \frac{y^{\rho-2}}{\log ^{3} y} d y\right| & \leqq \frac{x^{\beta-1}}{\log ^{2} x}+2 \int_{x}^{\infty} \frac{y^{\beta-2}}{\log ^{3} y} d y \\
& <\frac{x^{\beta-1}}{\log ^{2} x}+2 x^{\beta-1} \int_{x}^{\infty} \frac{d y}{y \log ^{3} y}=\frac{2 x^{\beta-1}}{\log ^{2} x}
\end{aligned}
$$

Hence

$$
\begin{aligned}
\left|\frac{1}{\rho} \int_{x}^{\infty} \frac{y^{\rho-2}(1+\log y)}{\log ^{2} y} d y\right| & <\frac{1}{|\rho(\rho-1)|} \frac{x^{\beta-1}}{\log x}+\frac{1}{|\rho-1|^{2}} \frac{2 x^{\beta-1}}{\log ^{2} x} \\
& <\frac{2+\log x}{\log ^{2} x} \cdot \frac{x^{\beta-1}}{\gamma^{2}} .
\end{aligned}
$$

$$
\sum_{\rho}\left|\frac{1}{\rho} \int_{x}^{\infty} \frac{y^{\rho-2}(1+\log y)}{\log ^{2} y} d y\right|<\frac{2+\log x}{\log ^{2} x} K(1, x) .
$$

So by Theorem 29 on p. 77 of Ingham [6], we have

$$
\left|\int_{x}^{\infty} \frac{(y-\psi(y))(1+\log y)}{y^{2} \log ^{2} y} d y\right|<\frac{2+\log x}{\log ^{2} x} K(1, x)+I,
$$


where

$$
\begin{aligned}
I & =\int_{x}^{\infty} \frac{\left(2 \log 2 \pi-\log \left(1-y^{-2}\right)\right)(1+\log y)}{2 y^{2} \log ^{2} y} d y \\
& <\frac{1+\log x}{2 \log ^{2} x} \int_{x}^{\infty} \frac{2 \log 2 \pi-\log \left(1-y^{-2}\right)}{y^{2}} d y \\
& =\frac{1+\log x}{2 \log ^{2} x}\left\{\frac{2 \log 2 \pi}{x}+\sum_{r=1}^{\infty} \frac{x^{-2 r-1}}{r(2 r+1)}\right\} \\
& <\frac{1+\log x}{\log ^{2} x}\left\{\frac{\log 2 \pi}{x}+\sum_{r=1}^{\infty} \frac{x^{-3}}{2 r(2 r+1)}\right\} \\
& <\frac{2+\log ^{2} x}{\log ^{2} x}\left\{\frac{\log 2 \pi}{x}+\frac{1-\log 2}{x^{3}}\right\} .
\end{aligned}
$$

From this, the lemma follows.

LEMMA 9. For $0 \leqq a<n$,

$$
\int_{x}^{\infty} y^{a-n-1} \frac{1+n \log y}{\log ^{2} y} d y \leqq \frac{n}{n-a} \cdot \frac{x^{a-n}}{\log x} .
$$

Proof. We have

Let us define

$$
\begin{aligned}
\int_{x}^{\infty} y^{a-n-1} \frac{1+n \log y}{\log ^{2} y} d y & =-\int_{x}^{\infty} y^{a} \frac{d}{d y}\left(\frac{y^{-n}}{\log y}\right) d y \\
& =\frac{x^{a-n}}{\log x}+a \int_{x}^{\infty} \frac{y^{a-n-1}}{\log y} d y \\
& \leqq \frac{x^{a-n}}{\log x}+\frac{a}{\log x} \int_{x}^{\infty} y^{a-n-1} d y \\
& =\frac{n}{n-a} \cdot \frac{x^{a-n}}{\log x} .
\end{aligned}
$$

$$
L(x)=\frac{2+\log x}{\log x}\left(K(1, x)+\frac{1.84}{x}+\frac{0.31}{x^{3}}\right)
$$

$$
+\frac{2.04}{x^{1 / 2}}+4.5 x^{-2 / 3}+\frac{1.02}{x-1}
$$

$$
M(x, a)=(\log x) \log \left(1+\frac{1}{2 \log ^{2} x}\right)-\frac{a}{\log x} .
$$

Lemma 10. If $1<A \leqq B$ and $a<\frac{1}{2}$ and

$$
\log A \geqq\left(\frac{1}{2} \cdot \frac{1+2 a}{1-2 a}\right)^{1 / 2}
$$

and $L(A)<M(B, a)$, then $L(x)<M(x, a)$ for $A \leqq x \leqq B$.

Proof. We readily verify that for $A \leqq x$ both $L(x)$ and $M(x, a)$ are decreasing functions of $x$. So for $A \leqq x \leqq B$ 


$$
L(x) \leqq L(A)<M(B, a) \leqq M(x, a) .
$$

Lemma 11. For $10^{8} \leqq x \leqq e^{600}$,

$$
L(x)<M(x, 0.31) .
$$

Proof. By (8.4) and (8.5) and by taking $K=\infty$ in Lemma 6, we find that

$$
L\left(10^{8}\right)<3 \times 10^{-4}<M\left(e^{600}, 0.31\right) .
$$

So we can use Lemma 10 with $A=10^{8}, B=e^{6 n 0}$.

LeMma 12 . For $e^{375} \leqq x$,

$$
L(x)<M(x, 0.47) .
$$

Proof. By taking $K=e^{25}$ in Lemma 6, we find that

$$
K(1, x)<0.0463 / x^{1 / 2}+e^{-9.61} x^{-1 /(25 R)}+10^{-10} .
$$

From this, we readily verify by (8.4) and (8.5) that

$$
\begin{aligned}
& L\left(e^{375}\right)<2.9 \times 10^{-5}<M\left(e^{1000}, 0.47\right), \\
& L\left(e^{1000}\right)<7 \times 10^{-6}<M\left(e^{4000}, 0.47\right), \\
& L\left(e^{4000}\right)<9 \times 10^{-9}<M\left(e^{3000000}, 0.47\right) .
\end{aligned}
$$

Then by three applications of Lemma 10, we verify (8.8) for $e^{375} \leqq x \leqq e^{3000000}$. Finally for $e^{3000000} \leqq x$, we take

and observe that

$$
K=\exp \frac{\log x}{R(\log \log x-\log 400)}
$$

$$
L(x)<0.028 / \log x<M(x, 0.47)
$$

by (8.4), Lemma 6 , and (8.5).

Lemma 13. For $10^{8} \leqq x$,

(8.9) $\left|\sum_{p \leqq x} \frac{1}{p}-\log \log x-B\right|<\log \left(1+\frac{1}{2 \log ^{2} x}\right)-\frac{1.02}{(x-1) \log x}$.

Proof. By (4.20), Lemma 8, and (3.39), we have

$$
\begin{aligned}
& \left|\sum_{p \leqq x} \frac{1}{p}-\log \log x-B\right| \\
& \leqq\left|\frac{\vartheta(x)-x}{x \log x}\right|+\left|\int_{x}^{\infty} \frac{(y-\psi(y))(1+\log y)}{y^{2} \log ^{2} y} d y\right| \\
& +\left|\int_{x}^{\infty} \frac{(\psi(y)-\vartheta(y))(1+\log y)}{y^{2} \log ^{2} y} d y\right| \\
& <\left|\frac{\vartheta(x)-x}{x \log x}\right|+\frac{2+\log x}{\log ^{2} x}\left(K(1, x)+\frac{1.84}{x}+\frac{0.31}{x^{3}}\right) \\
& +\int_{x}^{\infty} \frac{\left(1.02 y^{1 / 2}+3 y^{1 / 3}\right)(1+\log y)}{y^{2} \log ^{2} y} d y .
\end{aligned}
$$


We apply Lemma 9 with $n=1$, once with $a=\frac{1}{2}$ and once with $a=\frac{1}{3}$. Then, with the help of (8.4), the above inequality reduces to

$$
\left|\sum_{p \leqq x} \frac{1}{p}-\log \log x-B\right|<\left|\frac{\vartheta(x)-x}{x \log x}\right|+\frac{L(x)}{\log x}-\frac{1.02}{(x-1) \log x} .
$$

From this the lemma follows; we first assume $10^{8} \leqq x \leqq e^{375}$ and use Theorem 29, Lemma 11, and (8.5), and we then assume $e^{375} \leqq x$ and use Theorem 31, Lemma 12 , and (8.5).

We now proceed to the proof of Theorem 5 . It was proved for $x \leqq 16,000$ by the Rosser-Walker tabulation. For $16,000 \leqq x \leqq 10^{8}$, it follows from Theorem 20. Finally, for $10^{8} \leqq x$, it follows by Lemma 13 .

The proof of Theorem 6 proceeds similarly. For $10^{8} \leqq x$, it depends on a lemma analogous to Lemma 13 that uses Lemma 7 rather than Lemma 8. The entire proof parallels that of Theorem 5 so closely that we omit the details.

For $x \leqq 16,000$, Theorem 7 and Theorem 8 follow from the Rosser-Walker tabulation, and for $16,000 \leqq x \leqq 10^{8}$, they follow by Theorem 23 . So let us assume that $10^{8} \leqq x$. We apply Lemma 13 in the form that the left side of (8.9) with the absolute value bars removed is greater than the negative of the right side. In this, we substitute for $B$ from (2.7) and take the exponential of both sides. We get:

$$
\frac{e^{-c}}{\log x}\left(1+\frac{1}{2 \log ^{2} x}\right)>\left\{\prod_{p \leqq x}\left(1-\frac{1}{p}\right)\right\} \exp \left(\frac{1.02}{(x-1) \log x}+S\right)
$$

where

$$
S=\sum_{x<p}\left\{\log \left(1-\frac{1}{p}\right)+\frac{1}{p}\right\}=-\sum_{n=2}^{\infty} \frac{1}{n} \sum_{x<p} \frac{1}{p^{n}} .
$$

Taking $f(x)=x^{-n}$ in (4.13), we infer if $n>1$

$$
\sum_{x<p} \frac{1}{p^{n}}=-\frac{\vartheta(x)}{x^{n} \log x}+\int_{x}^{\infty} \frac{\vartheta(y)}{y^{n+1}} \cdot \frac{1+n \log y}{\log ^{2} y} d y .
$$

Then by Theorem 9 and Lemma 9

So

$$
\begin{aligned}
\sum_{x<p} \frac{1}{p^{n}} & <1.02 \int_{x}^{\infty} y^{-n} \frac{1+n \log y}{\log ^{2} y} d y \\
& \leqq \frac{1.02 n}{n-1} \cdot \frac{x^{1-n}}{\log x} \leqq \frac{1.02 n}{x^{n-1} \log x} .
\end{aligned}
$$

$$
S>-\sum_{n=2}^{\infty} \frac{1.02}{x^{n-1} \log x}=\frac{-1.02}{(x-1) \log x} .
$$

So by (8.10) we conclude that (3.26) holds. Inverting both sides gives (3.28).

Similarly, if we remove the absolute value bars on the left side of $(8.9)$ and take the exponential of both sides, we infer (3.29). Inverting both sides gives (3.25). 


\section{Proof of Theorem 15}

In this section, $\phi(n)$ again denotes the Euler totient function.

Lemma 14. If $m$ and $n$ are positive integers, and $n<\exp \vartheta\left(p_{m+1}\right)$, then

$$
n / \phi(n) \leqq \prod_{p \leqq p_{m}} p /(p-1) .
$$

Proof. Let $q_{1}, q_{2}, \cdots, q_{r}$ be the distinct primes, in increasing order, which divide $n$. Then

$$
\exp \vartheta\left(p_{m+1}\right)>n \geqq q_{1} \cdots q_{r} \geqq p_{1} \cdots p_{r}=\exp \vartheta\left(p_{r}\right) .
$$

So $r \leqq m$. Consequently

$$
n / \phi(n)=\prod_{s=1}^{r} q_{s} /\left(q_{s}-1\right) \leqq \prod_{s=1}^{r} p_{s} /\left(p_{s}-1\right) \leqq \prod_{s=1}^{m} p_{s} /\left(p_{s}-1\right),
$$

which is the same as (9.1)

By means of Lemma 14 we can verify (3.41) numerically for a succession of intervals if $\log n$ is not great. For instance, we readily verify numerically that

$$
\coprod_{p \leqq 7} p /(p-1)=4.375<e^{c} \log \log 210+5 /(2 \log \log 210) .
$$

So we can take $m=4$ in Lemma 14 , and conclude that if $210 \leqq n<2310=$ $\exp \vartheta\left(p_{m+1}\right)$, then

$n / \phi(n)<e^{C} \log \log 210+5 /(2 \log \log 210) \leqq e^{C} \log \log n+5 /(2 \log \log n)$

With the help of values of $\vartheta(x)$ and of

$$
\prod_{p \leqq x} p /(p-1)
$$

taken from the Rosser-Walker tabulation, we proceeded in a step-by-step manner as indicated above, verifying (3.41) for $3 \leqq n<\exp \vartheta(313)$ except at

$$
n=\prod_{p \leqq 23} p,
$$

at which point (3.42) holds. As $294<\vartheta(313)$, this verifies Theorem 15 for $n \leqq e^{294}$.

TheOREM 33. If $x \geqq 5$ and $n<\exp \vartheta(x)$, then

$$
n / \phi(n) \leqq \prod_{p \leqq x-2} p /(p-1) .
$$

Proof. Choose $m$ so that $p_{m+1} \leqq x<p_{m+2}$. Then $n<\exp \vartheta(x)=$ $\exp \vartheta\left(p_{m+1}\right)$. Also $5 \leqq p_{m+1}$, else we would have $p_{m+2} \leqq 5$, contradicting $5 \leqq x<p_{m+2}$. So $p_{m} \leqq p_{m+1}-2 \leqq x-2$. Now use Lemma 14 .

LEMMA 15. Let $n$ be an integer greater than unity and $y$ a real number such that $288 \leqq \log n+y$ and $\log n<\vartheta(\log n+y)$, and

$$
0 \leqq y-2 \leqq(0.9 \log n) / \log \log n .
$$

Then (3.41) holds for this value of $n$. 
Proof. By (3.29) and Theorem 33

$$
\begin{aligned}
\frac{e^{-c} n}{\phi(n)} & <\log (\log n+y-2)+\frac{0.5}{\log (\log n+y-2)} \\
& \leqq \log \log n+\log \left(1+\frac{y-2}{\log n}\right)+\frac{0.5}{\log \log n} \\
& \leqq \log \log n+\frac{y-2}{\log n}+\frac{0.5}{\log \log n} \\
& \leqq \log \log n+\frac{1.4}{\log \log n} .
\end{aligned}
$$

From this, (3.41) follows.

To complete the proof of Theorem 15, we have to show that (3.41) holds for $294 \leqq \log n$. In fact from Lemma 15 , one can deduce $(3.41)$ for $255 \leqq$ $\log n$. First assume $255 \leqq \log n \leqq 1340$. Take $y$ to be

$$
2+2(1+\log n)^{1 / 2} \text {. }
$$

Then we have $288<\log n+y<1420$. Also

$$
\log n=(\log n+y)-2(\log n+y)^{1 / 2},
$$

so that $\log n<\vartheta(\log n+y)$ by Theorem 19. As we easily verify that $0 \leqq y-2 \leqq(0.9 \log n) / \log \log n$, we infer (3.41) by Lemma 15. Finally, let $1340 \leqq \log n$. Take $y=(0.9 \log n) / \log \log n$. Then

$$
\log n<\vartheta(\log n+y)
$$

holds by (3.14), and the other hypotheses of Lemma 15 are readily verified, giving (3.41) again. 
TABLE I

$(1-\varepsilon) x<\psi(x)<(1+\varepsilon) x$ for $e^{b} \leqq x$

\begin{tabular}{|c|c|c|c|c|c|c|c|c|c|c|c|}
\hline$b$ & $m$ & $10^{n} \varepsilon$ & $n$ & $b$ & $m$ & $10^{n} \varepsilon$ & $n$ & $b$ & $m$ & $10^{n} \mathcal{\varepsilon}$ & $n$ \\
\hline 18.4 & 1 & 1.6327 & 2 & 62 & 4 & 1.3108 & 3 & 725 & 5 & 5.9384 & 4 \\
\hline 18.5 & 1 & 1.6295 & 2 & 64 & 4 & 1.2825 & 3 & 750 & 5 & 5.8017 & 4 \\
\hline 18.6 & 2 & 1.6256 & 2 & 66 & 4 & 1.2696 & 3 & 775 & 5 & 5.6682 & 4 \\
\hline 18.7 & 2 & 1.5987 & 2 & 68 & 4 & 1.2630 & 3 & 800 & 5 & 5.5378 & 4 \\
\hline 18.8 & 2 & 1.5722 & 2 & 70 & 4 & 1.2588 & 3 & 825 & 5 & 5.4103 & 4 \\
\hline 18.9 & 2 & 1.5462 & 2 & 72 & 4 & 1.2555 & 3 & 850 & 4 & 5.2843 & 4 \\
\hline 19.0 & 2 & 1.5206 & 2 & 74 & 5 & 1.2138 & 3 & 875 & 4 & 5.1397 & 4 \\
\hline 19.5 & 2 & 1.3993 & 2 & 76 & 5 & 1.1419 & 3 & 900 & 4 & 4.9991 & 4 \\
\hline 20.0 & 2 & 1.2880 & 2 & 78 & 5 & 1.1074 & 3 & 925 & 4 & 4.8624 & 4 \\
\hline 20.5 & 2 & 1.1861 & 2 & 80 & 5 & 1.0920 & 3 & 950 & 4 & 4.7294 & 4 \\
\hline 21 & 2 & 1.0928 & 2 & 85 & 5 & 1.0793 & 3 & 975 & 4 & 4.6001 & 4 \\
\hline 22 & 2 & 9.2933 & 3 & 90 & 5 & 1.0737 & 3 & 1000 & 4 & 4.4744 & 4 \\
\hline 23 & 2 & 7.9327 & 3 & 95 & 6 & 1.0075 & 3 & 1050 & 4 & 4.2331 & 4 \\
\hline 24 & 2 & 6.8090 & 3 & 100 & 6 & 9.9653 & 4 & 1100 & 4 & 4.0049 & 4 \\
\hline 25 & 2 & 5.8927 & 3 & 105 & 6 & 9.9194 & 4 & 1150 & 3 & 3.7703 & 4 \\
\hline 26 & 2 & 5.1592 & 3 & 110 & 6 & 9.8791 & 4 & 1200 & 3 & 3.5217 & 4 \\
\hline 27 & 2 & 4.5870 & 3 & 125 & 6 & 9.7608 & 4 & 1300 & 3 & 3.0730 & 4 \\
\hline 28 & 2 & 4.1548 & 3 & 150 & 6 & 9.5669 & 4 & 1400 & 3 & 2.6819 & 4 \\
\hline 29 & 2 & 3.8400 & 3 & 175 & 6 & 9.3768 & 4 & 1500 & 3 & 2.3411 & 4 \\
\hline 30 & 2 & 3.6192 & 3 & 200 & 6 & 9.1904 & 4 & 1600 & 2 & 2.0120 & 4 \\
\hline 31 & 2 & 3.4690 & 3 & 225 & 6 & 9.0078 & 4 & 1800 & 2 & 1.4334 & 4 \\
\hline 32 & 2 & 3.3691 & 3 & 250 & 6 & 8.8289 & 4 & 2000 & 2 & 1.0274 & 4 \\
\hline 33 & 2 & 3.3034 & 3 & 275 & 6 & 8.6535 & 4 & 2200 & 2 & 7.4229 & 5 \\
\hline 34 & 2 & 3.2601 & 3 & 300 & 6 & 8.4816 & 4 & 2400 & 2 & 5.4246 & 5 \\
\hline 35 & 2 & 3.2310 & 3 & 325 & 6 & 8.3131 & 4 & 2600 & 2 & 4.0328 & 5 \\
\hline 36 & 2 & 3.2110 & 3 & 350 & 6 & 8.1480 & 4 & 2800 & 2 & 3.0861 & 5 \\
\hline 37 & 2 & 3.1964 & 3 & 375 & 6 & 7.9861 & 4 & 3000 & 2 & 2.5073 & 5 \\
\hline 38 & 2 & 3.1853 & 3 & 400 & 6 & 7.8275 & 4 & 3200 & 3 & 2.4309 & 5 \\
\hline 39 & 3 & 2.9365 & 3 & 425 & 6 & 7.6721 & 4 & 3400 & 3 & 1.8801 & 5 \\
\hline 40 & 3 & 2.6399 & 3 & 450 & 6 & 7.5197 & 4 & 3600 & 3 & 1.4596 & 5 \\
\hline 42 & 3 & 2.2000 & 3 & 475 & 6 & 7.3704 & 4 & 3800 & 3 & 1.1388 & 5 \\
\hline 44 & 3 & 1.9387 & 3 & 500 & 6 & 7.2240 & 4 & 4000 & 3 & 8.9428 & 6 \\
\hline 46 & 3 & 1.8064 & 3 & 525 & 6 & 7.0806 & 4 & 4200 & 3 & 7.0899 & 6 \\
\hline 48 & 3 & 1.7467 & 3 & 550 & 6 & 6.9400 & 4 & 4400 & 3 & 5.7041 & 6 \\
\hline 50 & 3 & 1.7202 & 3 & 575 & 6 & 6.8023 & 4 & 4500 & 3 & 5.1602 & 6 \\
\hline 52 & 3 & 1.7072 & 3 & 600 & 6 & 6.6672 & 4 & 4600 & 3 & 4.7095 & 6 \\
\hline 54 & 3 & 1.6994 & 3 & 625 & 5 & 6.5182 & 4 & 4700 & 3 & 4.3563 & 6 \\
\hline 56 & 3 & 1.6936 & 3 & 650 & 5 & 6.3682 & 4 & 4800 & 3 & 4.1232 & 6 \\
\hline 58 & 4 & 1.5013 & 3 & 675 & 5 & 6.2216 & 4 & 4900 & 3 & 4.0977 & 6 \\
\hline 60 & 4 & 1.3740 & 3 & 700 & 5 & 6.0783 & 4 & 5000 & 3 & 4.9163 & 6 \\
\hline
\end{tabular}


TABLE II

\begin{tabular}{|c|c|c|c|c|}
\hline$x$ & $\vartheta(x)$ & $\sum_{p \leqq x} \frac{1}{p}$ & $\sum_{p \leqq x} \frac{\log p}{p}$ & $\prod_{p \leqq x} \frac{p}{p-1}$ \\
\hline 500 & $474.55444 \quad 41547$ & 2.0967095528 & 4.9444899600 & 11.1595015857 \\
\hline 1000 & $956.24526 \quad 51201$ & 2.1980801272 & 5.6095104754 & 12.3509756739 \\
\hline 1500 & 1462.1416518014 & 2.2556282528 & 6.0186961634 & 13.0829109945 \\
\hline 2000 & 1939.8392003026 & 2.2924484920 & 6.2932707024 & 13.5737500182 \\
\hline 2500 & 2433.6027529800 & 2.3210531990 & 6.5138437141 & 13.9677193817 \\
\hline 3000 & 2932.3592118787 & 2.3440493716 & 6.6958435999 & 14.2927053203 \\
\hline 3500 & 3409.4571845205 & 2.3622213278 & 6.8427532932 & 14.5548467736 \\
\hline 4000 & 3911.1453995812 & 2.3785830199 & 6.9772951026 & 14.7949800928 \\
\hline 4500 & 4412.1883105019 & 2.3927653465 & 7.0957103205 & 15.0063274987 \\
\hline 5000 & 4911.6953517069 & 2.4051886577 & 7.2008763227 & 15.1939385100 \\
\hline 5500 & 5391.3722383531 & 2.4158631315 & $7.29230 \quad 15305$ & 15.3570101674 \\
\hline 6000 & 5893.2974572481 & 2.4259840781 & 7.3798811065 & 15.5132405518 \\
\hline 6500 & 6408.9073671752 & 2.4354375880 & 7.4624921364 & 15.6606023831 \\
\hline 7000 & 6920.4210299437 & 2.4440157706 & 7.5381409953 & 15.7955297610 \\
\hline 7500 & 7364.8574160237 & 2.4509139779 & $7.59945 \quad 43711$ & 15.9048748550 \\
\hline 8000 & 7875.1503847974 & 2.4582917384 & 7.6655012982 & 16.0226587938 \\
\hline 8500 & 8343.9996634035 & 2.4646059355 & 7.7224305072 & 16.1241552820 \\
\hline 9000 & 8870.3749926578 & 2.4712444465 & 7.7826762189 & 16.2315579133 \\
\hline 9500 & 9418.3687733985 & 2.4777262760 & 7.8418745368 & 16.3371155421 \\
\hline 10000 & 9895.9913791570 & 2.4830599472 & 7.8908636043 & 16.4244896322 \\
\hline 10500 & 10403.9070475207 & 2.4884273950 & 7.9404300603 & 16.5128885620 \\
\hline 11000 & 10877.3416304695 & 2.4931702420 & 7.9844572244 & 16.5913963457 \\
\hline 11500 & 11362.4397133403 & 2.4977896786 & 8.0275503003 & 16.6682199665 \\
\hline 12000 & 11840.4857538722 & 2.5021224249 & 8.0681624606 & 16.7405988963 \\
\hline 12500 & $12348.83694 \quad 44657$ & 2.5065281281 & 8.1096367280 & 16.8145187368 \\
\hline 13000 & 12868.7280974239 & 2.5108452555 & 8.1504440734 & 16.8872689363 \\
\hline 13500 & 13371.7684532826 & 2.5148472175 & 8.1884274850 & 16.9549891186 \\
\hline 14000 & 13867.2925276925 & 2.5186272664 & 8.2244483834 & 17.0192034304 \\
\hline 14500 & $14307.28400 \quad 32521$ & 2.5218538317 & 8.2553102383 & 17.0742076210 \\
\hline 15000 & 14844.7916921653 & 2.5256530642 & 8.2917762756 & 17.1392020969 \\
\hline 15500 & 15384.2385636932 & 2.5293229775 & 8.3271281756 & 17.2022191093 \\
\hline 16000 & 15886.7924684213 & 2.5326250069 & 8.3590403921 & 17.2591170367 \\
\hline
\end{tabular}


TABLE III

\begin{tabular}{|c|c|c|c|}
\hline$n$ & $\psi(n)-\vartheta(n)$ & $n$ & $\psi(n)-\vartheta(n)$ \\
\hline 4 & $0.69314 \quad 71805 \quad 59945$ & 5041 & $90.52702 \quad 0408085442$ \\
\hline 8 & $1.3862943611 \quad 19891$ & 5329 & 94.817479849233834 \\
\hline 9 & 2.484906649788000 & 6241 & 99.186927701700855 \\
\hline 16 & 3.178053830347946 & 6561 & 100.285539990368965 \\
\hline 25 & $\begin{array}{llll}4.78749 & 17427 & 82046\end{array}$ & 6859 & 103.229978969535405 \\
\hline 27 & $5.88610 \quad 40314 \quad 50156$ & 6889 & $107.6488195773 \quad 32003$ \\
\hline 32 & $\begin{array}{lll}6.57925 & 12120 & 10101\end{array}$ & 7921 & $112.13745 \quad 5947064143$ \\
\hline 49 & $8.52516 \quad 13610 \quad 65414$ & 8192 & 112.830603127624088 \\
\hline 64 & $9.21830 \quad 8541625360$ & 9409 & $117.40531 \quad 41061 \quad 27471$ \\
\hline 81 & 10.316920830293469 & 10201 & 122.020434622968731 \\
\hline 121 & 12.714816103091840 & 10609 & 126.655163611198366 \\
\hline 125 & 14.324254015525940 & 11449 & 131.327992445660272 \\
\hline 128 & $\begin{array}{ll}15.01740 & 1196085886\end{array}$ & 11881 & $136.01934 \quad 03278 \quad 89416$ \\
\hline 169 & 17.582350553547422 & 12167 & $139.15483 \quad 4543818566$ \\
\hline 243 & 18.680962842215532 & 12769 & 143.882222362530906 \\
\hline 256 & $19.3741100227 \quad 75477$ & 14641 & 146.280117635329277 \\
\hline 289 & 22.207323366831693 & 15625 & $147.88955 \quad 5547763377$ \\
\hline 343 & $24.15323 \quad 3515887007$ & 16129 & $152.73374 \quad 26342 \quad 21969$ \\
\hline 361 & $27.09767 \quad 24950 \quad 53447$ & 16384 & 153.426889814781914 \\
\hline 512 & $27.7908196756 \quad 13392$ & 16807 & $155.3727999638 \quad 37227$ \\
\hline 529 & 30.926313891542542 & 17161 & $160.24799 \quad 7287038379$ \\
\hline 625 & $32.53575 \quad 1803976642$ & 18769 & 165.167978212866504 \\
\hline 729 & 33.634364092644752 & 19321 & 170.102452145997195 \\
\hline 841 & 37.001659922631226 & 19683 & $171.2010644346 \quad 65305$ \\
\hline 961 & $40.4356471271 \quad 16372$ & 22201 & $176.20501 \quad 07406 \quad 10764$ \\
\hline 1024 & 41.128794307676318 & 22801 & 181.222290577425689 \\
\hline 1331 & 43.526689580474688 & 24389 & 184.589586407412163 \\
\hline 1369 & $47.1376074931 \quad 18913$ & 24649 & $189.64583 \quad 22127 \quad 60471$ \\
\hline 1681 & $50.8511795598 \quad 23221$ & 26569 & 194.739582413567233 \\
\hline 1849 & 54.612379675516783 & 27889 & $199.85757 \quad 6225983988$ \\
\hline 2048 & 55.305526856076728 & 28561 & $202.4225255834 \quad 45525$ \\
\hline 2187 & 56.404139144744838 & 29791 & 205.856512787930671 \\
\hline 2197 & $58.96908 \quad 8502206375$ & 29929 & 211.009804382428450 \\
\hline 2209 & $62.81923 \quad 61039 \quad 16433$ & 32041 & 216.197190188269205 \\
\hline 2401 & $64.76514 \quad 6252971747$ & 32761 & 221.395687219535031 \\
\hline 2809 & $68.73543 \quad 8166523868$ & 32768 & 222.088834400094976 \\
\hline 3125 & $70.34487 \quad 6078957969$ & 36481 & 227.341107828141606 \\
\hline 3481 & $74.42241 \quad 3522863688$ & 37249 & 232.603798017046492 \\
\hline 3721 & 78.533287387037000 & 38809 & $\begin{array}{llll}237.88700 & 17457 & 84480\end{array}$ \\
\hline 4096 & $79.22643 \quad 4567596945$ & 39601 & 243.180306570508972 \\
\hline 4489 & 83.431127186987911 & 44521 & $248.53216 \quad 4703985039$ \\
\hline 4913 & $86.26434 \quad 0531044127$ & 49729 & $253.93933 \quad 64754 \quad 45158$ \\
\hline
\end{tabular}


TABLE IV

\begin{tabular}{|c|c|c|c|c|}
\hline$n$ & & $-\zeta^{\prime}(n)$ & $-\zeta^{\prime}(n) / \zeta(n)$ & $\sum_{p} p^{-n} \log p$ \\
\hline 2 & 0.93754 & $82543 \quad 15843 \quad 75$ & $\begin{array}{llll}0.56996 & 09930 & 94532 & 80\end{array}$ & $\begin{array}{ll}0.49309 & 11093 \quad 68764 \quad 43\end{array}$ \\
\hline 3 & 0.19812 & 624288563685 & $0.1648226821 \quad 5827724$ & 0.15075755554395043 \\
\hline 4 & 6891 & 126589612538 & $\begin{array}{l}63669764955371 \quad 13\end{array}$ & 6060763335077008 \\
\hline 5 & 2857 & 378050946295 & $2755 \quad 619219153047$ & $\begin{array}{lllll}2683 & 86012 & 76798 & 36\end{array}$ \\
\hline 6 & 1285 & 216513179572 & 1263306903251106 & 1245908072280000 \\
\hline 7 & 603 & 351696087564 & 598355857063840 & 594068903914820 \\
\hline 8 & 290 & 195255371067 & $\begin{array}{llll}289 & 01683 & 08046 & 75\end{array}$ & $\begin{array}{llll}287 & 95247 & 08729 & 24\end{array}$ \\
\hline 9 & 141 & 598222724181 & 141314407881170 & 141049192142453 \\
\hline 10 & 69 & $\begin{array}{lll}70330 & 08171 & 39\end{array}$ & $\begin{array}{llll}69 & 63404 & 45284 & 02\end{array}$ & $\begin{array}{llll}69 & 56784 & 47344 & 62\end{array}$ \\
\hline 11 & 34 & 502222236836 & $\begin{array}{llll}34 & 48518 & 00538 & 42\end{array}$ & 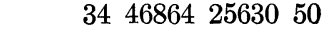 \\
\hline 12 & 17 & $1382845854 \quad 35$ & $171340681216 \quad 67$ & $1712993 \quad 5244621$ \\
\hline 13 & & 532390865593 & $\begin{array}{llll}8 & 53134 & 39558 & 17\end{array}$ & $85303109167 \quad 11$ \\
\hline 14 & & 254149338178 & 4253888795423 & 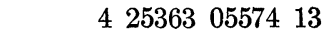 \\
\hline 15 & & 123108553300 & 2123043613140 & 2122979056275 \\
\hline 16 & & 060244203251 & $\begin{array}{lll}106022 & 8000572\end{array}$ & $106021 \quad 1861676$ \\
\hline 17 & & $52968 \quad 83357 \quad 53$ & $52968 \quad 42904 \quad 49$ & $5296802557 \quad 64$ \\
\hline 18 & & $2647002978 \quad 82$ & $2646992874 \quad 47$ & 264698278780 \\
\hline 19 & & 132302369478 & $13230 \quad 2117017$ & 132301864851 \\
\hline 20 & & 66135302074 & 66135238983 & $6613 \quad 5175942$ \\
\hline 21 & & 33062367677 & 33062351908 & $3306 \quad 23361 \quad 48$ \\
\hline 22 & & 16529425416 & 16529421475 & 16529417535 \\
\hline 23 & & 8264127238 & 8264126253 & 8264125268 \\
\hline 24 & & 4131868629 & 4131868383 & 4131868137 \\
\hline 25 & & 2065869360 & 2065869298 & 2065869236 \\
\hline 26 & & 1032913038 & 1032913023 & 1032913008 \\
\hline 27 & & 516449308 & 516449304 & 516449300 \\
\hline 28 & & 258222251 & 258222250 & $25 \quad 82222 \quad 49$ \\
\hline 29 & & 129110325 & 129110324 & 129110324 \\
\hline
\end{tabular}




\section{BibLIOGRAPHY}

1. C. L. Baker and F. J. Gruenberger, The first six million prime numbers, Microcard Foundation, West Salem, Wisconsin, 1959.

2. H. T. DAvis, Tables of the higher mathematical functions, Vol. II, Bloomington, Indiana, The Principia Press, Inc., 1935.

3. P. Finsler, Über die Primzahlen zwischen $n$ und $2 n$, Festschrift zum 60 . Geburtstag von Prof. Dr. Andreas Speiser, Zürich, Orell-Füssli, 1945, pp. 118-122.

4. J. W. L. GLaisher, Table of the values of $\frac{1}{2} \cdot \frac{2}{3} \cdot \frac{4}{5} \cdots \frac{x-1}{x}$, the denominators being the series of prime numbers, Messenger of Mathematics, vol. 28 (1898), pp. 1-17.

5. J. P. Gram, Undersфgelser angaaende Maengden af Primtal under en given Graense, K. Danske Vidensk. Selskabs Skrifter, Naturv. og Math. Afd., ser. 6, vol.2 (1881-86), pp. 183-308.

6. A. E. Ingham, The distribution of prime numbers, Cambridge Tract No. 30, Cambridge University Press, 1932.

7. E. Landau, Handbuch der Lehre von der Verteilung der Primzahlen, 2 vols., Leipzig, Teubner, 1909. Reprinted in 1953 by Chelsea Publishing Co., New York.

8. D. H. Lenmer, On the roots of the Riemann zeta-function, Acta Math., vol. 95 (1956), pp. 291-298.

9. - - Extended computation of the Riemann zeta-function, Mathematika, vol. 3 (1956), pp. 102-108.

10. D. N. Lehmer, List of prime numbers from 1 to 10,006,721, Carnegie Institution of Washington, Publication No. 165, 1914.

11. Barkley Rosser, The $n$-th prime is greater than $n \log n$, Proc Lond. Math. Soc. (2), vol. 45 (1939), pp. 21-44.

12. - Explicit bounds for some functions of prime numbers, Amer. J. Math., vol. 63 (1941), pp. 211-232.

13. J. BARKLEy Rosser, Explicit remainder terms for some asymptotic series, Journal of Rational Mechanics and Analysis (J. Math. Mech.), vol. 4 (1955), pp. 595-626.

14. P. Tchebichef, Mémoire sur les nombres premiers, J. Math. Pures Appl. (1), vol. 17 (1852), pp. 366-390.

15. E. Trost, Primzahlen, Basel, Birkhäuser, 1953.

16. A. WAlther, Anschauliches zur Riemannschen Zetafunktion, Acta Math., vol. 48 (1926), pp. 393-400.

17. I. M. Vinogradov, Novaya ocenka funkcii $\zeta(1+i t)$, Izv. Akad. Nauk SSSR, Ser. Mat., vol. 22 (1958), pp. 161-164.

18. Tables of sine, cosine and exponential integrals, 2 vols., W.P.A. Tables prepared in 1940 under the sponsorship of the National Bureau of Standards.

19. Kenneth I. Appel and J. Barkley Rosser, Table for estimating functions of primes, IDA-CRD Technical Report Number 4, September, 1961 (available on request from Communications Research Division, Institute for Defense Analyses, von Neumann Hall, Princeton, New Jersey).

Institute for Defense Analyses

Princeton, New Jersey

Pennsylvania State University

University Park, Pennsylvania 